\title{
EFFICIENT METHOD TO IMPROVE HUMAN BRAIN SENSOR ACTIVITIES USING PROPOSED NEUROHEADSET DEVICE EMBEDDED WITH SENSORS: A COMPREHENSIVE STUDY
}

\author{
Rajib Chowdhury, A F M Saifuddin Saif \\ Department of Computer Science \\ Faculty of Science and Information Technology \\ American International University - Bangladesh \\ saif@aiub.edu
}

\begin{abstract}
The main purpose of this research is to investigate the human brain sensor activities related prior researches towards the needs of an efficient method to improve the human brain sensor activities. Human brain activities mainly measured by brain signal acquired from the brain sensor electrodes positioned on several parts of the brain cortex. Although previous researches investigated human brain activities in various aspects, the improvement of the human brain sensor activities is still unsolved. In today's world, it is very crucial need for improving the sensor activities of the human brain using that human brain improved signal externally. This research demonstrated a comprehensive critical analysis of human brain activities related prior researches to claim for an efficient method integrated with proposed neuroheadset device. This research presented a comprehensive review in various aspects like previous methods, existing frameworks analysis and existing results analysis with the discussion to establish an efficient method for acquiring human brain signal, improving the acquired signal and developing the sensor activities of the human brain using that human brain improved signal. Demonstrated critical review has expected for constituting an efficient method to improve the performance of maneuverability, visualization, subliminal activities and so forth on human brain activities.
\end{abstract}

Keywords: Human Brain Activities; Human Brain Signal; Efficient Method; Proposed Neuroheadset Device; Brain Engineering;

\section{INTRODUCTION}

One of the most complex things which have discovered in the universe is the human brain. In every stage of life, people face everything using brain (Rao, Lakshmi, and Prasad 2012). In the neuroscience research, imaging of human brain activity is highly required for the better understanding of the human brain works (Taruno et al., 2013). It works like a network that passes the information's to several regions of the body parts and also controls the entire functions of the body. It has a significant contribution to receive, store and pass information (Sulaiman, Cheng Chee, Hadi, Mustafa, and Jadin 2014). Brain signal helps to define the activity of the human brain from the scalp.

The human brain can losses the efficiency when it feels to get tired and also feels anxious for the several types of worst proceedings. The human brain competence can easily decrease by the sentiment that causes disability to make the best assessments and also the reason for reducing the capability of thinking. Age can be another reason to 
increases the complexities in the human brain function. In artificial intelligence research, the improvement of the human brain activities will be a great challenge.

In previous, researchers have established emotion recognition; human brainmachine interactions; wheelchair control; human brain auditory activity observation; human thought interpretation; brain-computer interfaces; etc. using the human brain activity as well as the human brain signal. This research actually demonstrates the exploration of human brain sensor activities related previous research for establishing an efficient method assists to improve the human brain activities using proposed neuroheadset device embedded with sensors. In the future, we will illustrate the details framework helps to improve the human brain activities. This framework will be constituted using several steps like brain signal acquisition, acquired signal processing, signal feature extraction, the brain signal improvement and also the human brain activities improvement. The complete procedure will continue in a proposed neuroheadset device with the help of the efficient method. This efficient method will be constituted using several methods like signal acquisition technique, signal processing method, signal improvement method and also brain activity development approach.

\section{RESEARCH BACKGROUND}

Rushambwa and Mythili (2017) focused on the effects of mental and physical subliminal behavior on the human brain signal. For this, they used an EEG frontal lobe sensor, a Neurosky brain wave sensor with an ear clip for grounding, and a headband for acquiring the EEG signals. Then, they deducted the voltage from the brain through common mode rejection and also changed into a mono channel EEG signal. After that, they extracted the values of poor signal quality, attention, and meditation using FFT from the serial stream of the encoded values which was sent out by the brainwave and that also appeared as a serial stream of bytes using the standard Bluetooth serial port profile (SPP). They also configured the HC05 Bluetooth module using Cool term software through AT commands to receive the data wirelessly from the brainwave sensor and passed it via UART to the controller. Finally, they implemented Hardware circuitry in Proteus software (Rushambwa and Mythili 2017).

R. Majid Mehmood, Du, and Lee (2017) recognized the emotion using maximum characteristics of features and methods which extracted from deep learning research domains. They recorded and preprocessed EEG data using the EMOTIV-EPOC system and the EEGLAB toolbox respectively. After that, they measured the signal activity in the time series data using Hjorth parameters for various frequency ranges. Then, they used a balanced one-way ANOVA method for selecting the features of optimal EEG. The optimal EEG features were further processed to classify the emotion (R. Majid Mehmood, Du, and Lee 2017).

Wijayasekara and Manic (2013) used an Emotiv EPOC headset to measure brain activity. At first, they concentrated on raise the conductivity of the contact pad. For this, they considered a saline solution to hydrate an electrode of the 14 sensors. These sensors have positioned according to the international 10-20 system. Then, processed signals were combined from the Emotiv SDK using implemented GUI in the C++ environment and controlled the outputs to the mobile robot. They also identified the desired action and facilitated the training of the system to different subjects using Emotiv SDK. They also converted the action into motor speeds which helped to control the differential Wheel Robot. Finally, they used Lego NXT to create contact between a computer and the robot wirelessly through Bluetooth (Wijayasekara and Manic 2013). 
Shuangquan, Jie, Ningjiang, Xin, and Qinfeng (2005) recognized the human activities using tri-axial accelerometers, through matching the patterns of the objects movement's used for detecting and transforming the changes in capacitance into an output voltage of the analog system. Here, an onboard A/D converter was used to digitize this output voltage and contacted through a Serial Peripheral Interface (SPI). The KXP74 accelerometer was used to collect the data of acceleration. They mounted the sensors on the board and then sealed it hermetically. They also attached node of a sensor wirelessly that transmitted the data to the base station via the RF signal. They used a linear model for describing the mapping from electrical data to acceleration. They extracted the frequency-domain entropy, mean, energy, acceleration, standard deviation, and the correlation between axes. They also used Decision Tree (DT) C4.5, Support Vector Machine (SVM), and Multiple-Layer Perception (MLP) neural networks for performing the activities of recognition. Finally, they developed the data in a centralized way in the application of almost all recognition (Shuangquan, Jie, Ningjiang, Xin, and Qinfeng 2005).

Suzuki, Suzuki, Baba, and Furuta (2005) focused on the characteristics of human in machine operation. In here, they concentrated on the pendulum graphics where the physical rule was obeyed using the motion changes by the forces of operator's. Then, they sent out information to the human high dimensional function from the environment and a machine. They adopted information on force sense in a part of interfaces of the physical man-machine system to increase the maneuverability. They introduced the Haptic Test to detect the visual information, the Virtual Internal Model (VIM) for the slider control and the Near-Infrared Spectroscopy (NIRS) to detect changes which correspond with the activation of neural in the brain region. VIM behaved like a virtual model with particular properties of mass, stiffness, and damping to find haptic sense information (Suzuki, Suzuki, Baba, and Furuta 2005).

\section{PREVIOUS METHODS AND ANALYSIS}

The brain activities usually monitored by observing the electrical signals produced in the neurons (Arman, Ahmed, and Syed 2012). In many cases, researchers concentrated on the processing of the human brain signal to solve their identified problems. For signal processing, they followed several steps like signal acquisition, preprocessing, feature extraction and classification. In every phase, they considered an individual method to solve their identified problem. The main aim of us is to develop the human brain activities by processing the brain signal. For that, we have to concentrate on existing brain signal processing methods. We have demonstrated the comparison of previous methods based on the approaches which were previously established by the researcher for the brain signal processing in below:

Brain activities can be measured from the brain signal acquired by using the invasive or the non-invasive technique (Rao et al., 2012). Inspect of Invasive signal acquisition techniques provide excellent signals quality, it requires a surgery which is an ethical controversy. For this, most of the case, researchers focused on Non-Invasive acquisition method using functional Magnetic Resonance Imaging (fMRI), ElectroEncephalography (EEG), Magneto Encephalography (MEG), etc. In the Neuro Port data acquisition system, researcher trialed each electrode at $30 \mathrm{kHz}$ and pertained an analog at $0.3 \mathrm{~Hz}$ to $7.5 \mathrm{kHz}$ band-pass filter to each electrode (Even-Chen et al., 2018). Compared to conventional disc electrode signals, Tri-polar concentric ring electrode (TCRE) sensors EEG has better reciprocal information, better signal to noise ratio, and spatial resolution than others (Besio 2017). 

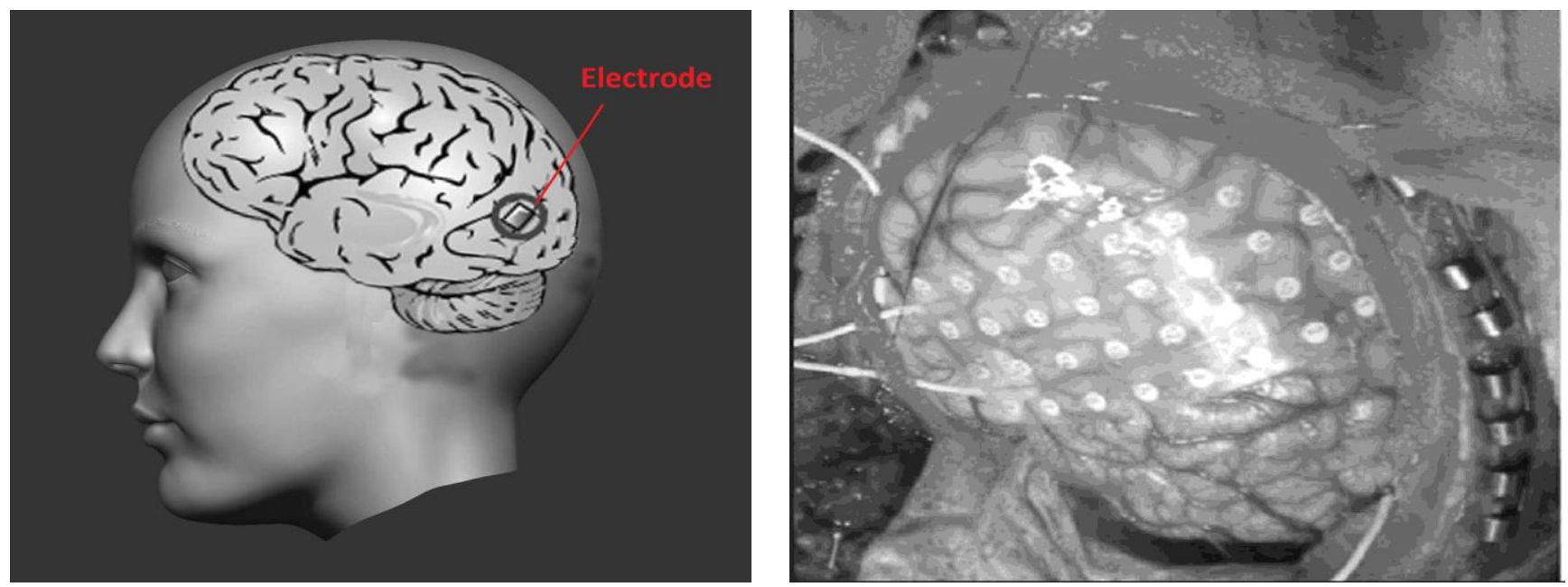

Figure 1. Electrode Placement System for Invasive Signal Acquisition Technique

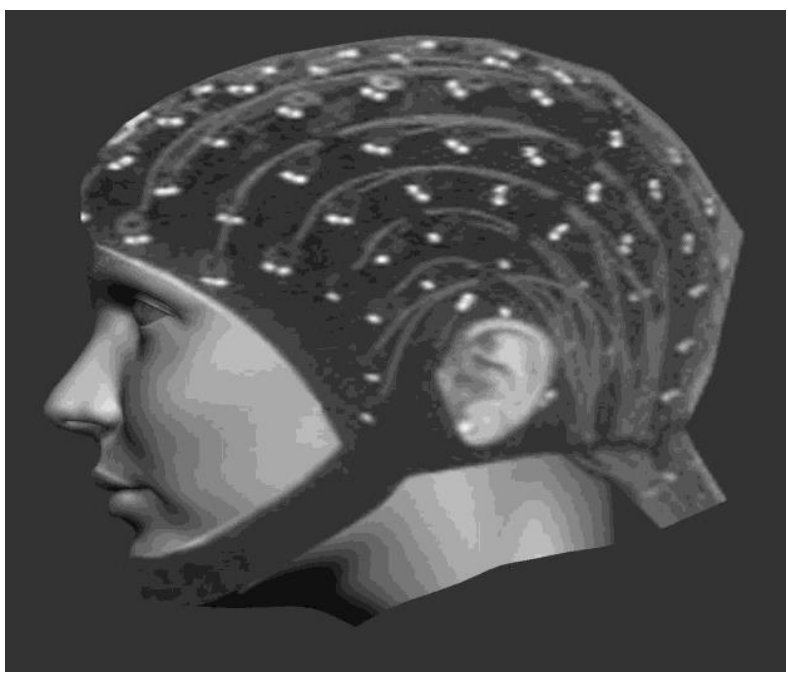

Figure 2. Electrode Placement System for Non- Invasive Signal Acquisition Technique

In previous, EEG has frequently used due to its simplicity and easiness of use which helped to meet the BCI specifications in real time implementation (Rao et al., 2012). It has a high temporal resolution, safety, and easiness technique but it has low spatial resolution and nonstationary signal (Lakshmi et al., 2014). Tri-polar concentric ring electrode (TCRE) sensors can use to acquire various bio-signals (Besio 2017). Strong visual evoked potentials also can see in the tEEG.

In most of the scenario, acquired brain signals were infected using various noise and artifacts. Principal Component Analysis (PCA) helps to classify data and helps to decrease of feature dimensions. In a BCI system, despite good classification results of the PCA, the ICA performs better than PCA (Lakshmi et al., 2014).

Independent component analysis (ICA) demonstrated a significant role in decomposing mixed data in independent components (ICs) when deriving RSNs from 
EEG/MEG data (Li, Yuan, Urbano, Cha, and Ding 2017). ICA showed routine for large-sized data, computationally proficient. It can decompose the signal data into temporally independent components and spatial specific components (Lakshmi et al., 2014).

Independent Component Analysis (ICA), Principal Component Analysis (PCA), and Fast Fourier Transformations (FFT) were mostly used to extract the features from the signal (Lakshmi et al., 2014). ICA was used to remove artifacts from independent signals and also contributed to separate noise for eye movement (Li, Yuan, Urbano, Cha, and Ding 2017). It can help to recognize the brain signals but it requires more computations for decomposition (Lakshmi et al., 2014). FFT is an influential method for frequency analysis and also helps to transfer the time domain data into power values in the frequency domain, but it is only appropriate for stationary signals and linear random methods. It suffers from noise, and also shows inappropriate for poor time localization in respect of various kinds of applications (Gutmann et al., 2015).

PCA is one of the exploratory analyses of multivariate data to investigate and deduct the dimensionality of data without loss of important information (Lakshmi et al., 2014; Suzuki et al., 2005). It holds lack of redundancy, helps to reduce the complication in images grouping and helps to decrease the noise for the maximum variation. In the case of PCA, only the trainee images, which are the representation of a smaller database, were also stored in the form of the researchers' protrusion (Karamizadeh, Abdullah, Manaf, Zamani, and Hooman 2013).

For classification Lakshmi, Prasad, and Prakash (2014) used various types of classifiers such that Artificial Neural Networks (ANN) based classifiers, linear classifiers, Nearest Neighbor classifiers and Nonlinear Bayesian classifiers (NBC). Support Vector Machine (SVM) and Linear Discriminant Analysis (LDA) were most commonly used to classify the data. Comparing with previous research, although, LDA demonstrates less computational requirements, easy to use and higher efficiency in experimental results for accuracy, LDA fails when the discriminatory function is not in the mean but invariance of the features. For non-Gaussian distributions, it may not preserve the complex structures. SVM provides good simplification and performance result, which is higher than other linear classifier but it has high computational complexity. Minimum-Redundancy-Maximum-Relevance (mRMR) method helps to choose the features that associates with the classification variable appropriately, reduce redundancy between bad and good features using Mutual Information (MI) methods (Atkinson and Campos 2016). ANN is simple to use and execution, robust in nature, able to easy computations. It requires a small training set, but it is not able to build easily. Its performance also depends on the number of neurons in the hidden layer (Lakshmi et al., 2014). Recurrent Neural Network (RNN) is able to use their internal memory by calculating the data for both forward and backward direction with two separate hidden layers which have loaded towards the same output layer. Deep Neural Network (DNN) helps to facilitate the learning process and amalgamate the Context. It can also perform as Semantic and Sentiment analyzer. DNN generally perform as feed forward networks where data can able to flow from input to output without following any backtracking (Hebbar 2017). NBC needs the small amount of training data to calculate the constraints data approximately, compute the variance of class variables. It has no need to determine the whole covariance matrix without good approximation for the class possibilities accurately (Lakshmi et al., 2014). In another side, the OSEC_111 algorithm is faster than SVM and NN but slower than Naïve Bayes and k-NN classifier. Quadratic Discriminant Analysis (QDA) and k-NN provide comparatively better classification findings than SVM (Atkinson and Campos 2016). 


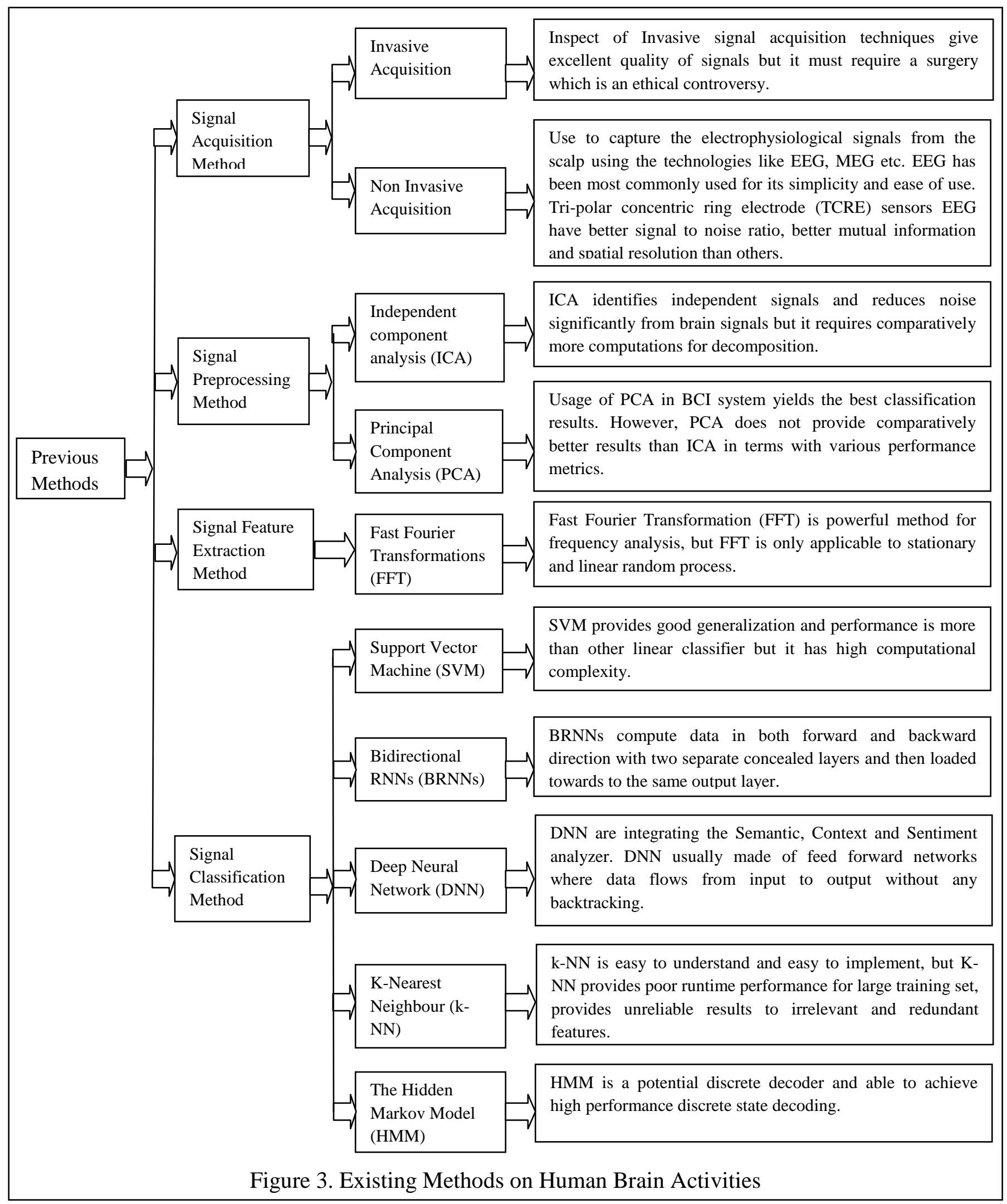

K-Nearest Neighbour (k-NN) classifier is a feature vector and also a nonparametric classifier. It helps to recognize very easily, execute and debug. The runtime performance of $\mathrm{k}-\mathrm{NN}$ is poor if the training data set is large, perceptive to inappropriate 
and also contains redundant features, on complicated classification tasks outperformed by other classification techniques (Lakshmi et al., 2014). The Hidden Markov Model (HMM) is a prospective discrete state decoder that would be capable of obtaining the higher level performance of discrete state decoding (Kao, Nuyujukian, Ryu, and Shenoy 2017).

From the above review based on the existing methods, we observed that NonInvasive EEG signal acquisition technique was highly used to acquire the human brain signal. Independent Component Analysis performed better to lessen the artifacts and noise from the signal data. Fast Fourier Transformation was mostly utilized to reduce the poor signal quality. In previous, more classification techniques were used to classify the signal data, but there is no existing improvement method to improve the signal. There is also no improvement approach exist for the development of human brain activities.

\section{EXISTING FRAMEWORKS AND ANALYSIS}

Previous methods were applied to solve the researchers identified problems for each phase of the established framework. They, at first, established a framework with the required methods for their research to solve their identified problem in the experiment level. In this section, we reviewed the previous research framework based on the sensor activities of the human brain. In previous, the human brain sensor activities related more researches have done based on the researcher's established framework. We have demonstrated the review of existing frameworks based on human brain activities in below:

Neurally and ocularly informed graph-based models were designed to search 3D environments with a represented framework. In here, at first, EEG data, pupil area data, and eye position data have collected in random route traversal. Then, Sensorium DBPA1 Amplifier was used to amplify the EEG data. $77 \mathrm{Ag} / \mathrm{AgCl}$ electrodes were also considered to collect the data. After that, an Eye Link 1000 eye tracker was used to collect eye position and pupil area data from one eye. In next, Principal Component Analysis was considered to reduce the dimensionality of the feature space and avoid rank deficiency issues. The Temporal independent component analysis was used to identify components whose temporal patterns of activity were statistically independent of one another. Within-Bin Classification and Cross-Bin Classification were applied to classify the data. The Final search has done after Label Self-Tuning and Route Planning (TSP Solver). In here, EEG gave independent information individually to allow a hybrid classifier to generate more precise output, but the addition of an eye tracker in a BCI system may induce material and calibration costs (Jangraw, Wang, Lance, Chang, and Sajda 2014).

SVM classifier and Hjorth Parameters were considered to recognize the emotion based on the acquired EEG signal with an established framework. In here, researchers, at first, acquired the EEG signal data using non-invasive signal acquisition. Then, they recorded, preprocessed and featured extracted the EEG signal data. Hjorth Parameters was also used to convert EEG data in the time-frequency domain. Finally, SVM classifier was used to classify the emotion recognition. In here, SVM classifier helped to obtain the highest 70\% accuracy for recognizing the emotion (Raja Majid Mehmood and Lee 2015).

Design and implementation of Brain-Computer Interface for wheelchair control were established using a recognized framework. This framework was constituted using 
several steps like EEG signal acquisition, preprocessing, feature extraction, translate into specified command and control wheelchair movement using the command. In here, the main advantage is that the average success rate is about $80 \%$ for controlling the wheelchair (Turnip, Suhendra, Esti, and Sihombing 2017).

Brain signals classification in a rapid sequential visual appearance task was improved using Active Learning (AL) has been established with a constitutional framework. At first, researchers have recorded and analyzed the EEG signal data, and then, they classified the signal data using Hierarchical Discriminant Component Analysis (HDCA), Common Spatial Patterns (CSP), XDawn + Bayesian Linear Discriminant Analysis (XDBLDA), Confidence, Merging Classifier Output, and CrossValidation (CV). Finally, they used the Active Learning and completed the statistical analysis. Here, some additional information helped to estimate a better decision boundary (Marathe, Lawhern, Wu, Slayback, and Lance 2016).

A compact and autoclavable system was demonstrated using researchers established framework. This system was used to record the keen extracellular neural data and monitor the human brain pressure. Then, they introduced a headstage design and the system control unit design. Headstage design was included using mechanical design, integrated amplifiers, and the pressure sensor. Headstage embedded with a strain-gauge based pressure sensor in order to give the medical surgeon for measuring and controlling the pressure exerted on the brain function during recordings. The system control unit design embedded using the neural data processing system and head stage positioning control system (Angotzi et al. 2015).

Spatial resolution improvement of EEG source reconstruction using swLORETA was build using researchers established framework. In here, the findings, based on sLORETA and swLORETA, helped to localize the dipole's activity from the EEG signal was introduced using semi-synthetic data (Boughariou, Jallouli, Zouch, Slima, and Hamida (2015).

From the above review based on the framework, we observe that signal acquisition, processing, and classification based frameworks were mostly used to solve the researchers identified problems. So, in this research, we focused on methods based on signal acquisition, processing, and signal improvement. But there is no existing method to improve brain activities.

\section{EXPERIMENTAL ANALYSIS AND DISCUSSION}

In this section, we reviewed the previous research experiment based on the sensor activities of the human brain for identifying the best methods which perform better to get the experimental highest result. In previous, human brain sensor activities related more research have done based on the experimental analysis.

Thirty young males were appointed as individuals in the experiment for emotion reorganization based on EEG signal using the SVM classifier. The EEG signals were acquired using the 10/20 internationally recognized placement system and were also recorded through Brain-Vision System using 18 electrodes. In here, EEGLAB Toolbox was considered to preprocess the signals. Hjorth Parameters has been used to convert the EEG data into a time-frequency domain. SVM classifier was used to classify the emotion recognition. The highest 70 percent accuracy was obtained using SVM for two emotions shown in Table 1 (Raja Majid Mehmood and Lee 2015).

Experiments used information on 14 channels while DEAP data includes EEG based signals extracted from a 32-channel BCI device. GA-SVM is other state-of-the-art 
features selection method used to compare with mRMR in order to tune the model. Three kernel configurations were tested based on the SVM using different kernel functions and degrees. Strength estimation of candidate solutions in the GA has utilized the classification accuracy of the SVM. The results showed that the mRMR-based feature selection method performed the most significant feature selection strategy than GA-SVM for Arousal and Valence dimensions. The accuracy results have shown in Table 2 (Atkinson and Campos 2016).

Table 1. Accuracy Results of the Emotion Groups with SVM Classifier

\begin{tabular}{|c|c|c|}
\hline Emotion Groups & Emotion Contains & $\begin{array}{c}\text { Accuracy }(\%) \text { with SVM } \\
\text { classifier }\end{array}$ \\
\hline $\mathbf{E 5}$ & $\begin{array}{l}\text { Five Emotions including Calm, } \\
\text { Happy, Neutral, Scared and Sad. }\end{array}$ & 30 \\
\hline $\mathbf{E 4}$ & $\begin{array}{c}\text { Four Emotions including Calm, } \\
\text { Happy, Scared and Sad. }\end{array}$ & 45 \\
\hline $\mathbf{E 3}$ & $\begin{array}{c}\text { Three Emotions including Happy, } \\
\text { Neutral and Sad. }\end{array}$ & 55 \\
\hline E2 & $\begin{array}{c}\text { Two Emotion including Happy } \\
\text { and Sad }\end{array}$ & 70 \\
\hline
\end{tabular}

Table 2. Accuracy Results for Arousal and Valence Dimension with MRMR and GASVM Methods

\begin{tabular}{ccc}
\hline Method Name & Accuracy (\%) for Arousal & Accuracy (\%) for Valence \\
\hline mRMR & 60.72 & 62.39 \\
GA-SVM & 56.69 & 53.46 \\
\hline
\end{tabular}

Classifier Trainer has run with the corresponding data obtained as input; the circumstance read through the data while feature vectors were extracted and write them all into one pattern file for using it later. SVM was used to classify the data. They also executed the testing step by running mi-CSP-4-online.xml in Open Vibe with adjusting simple DSP filter by $\mathrm{x}-0.5$. From the overall result, the percentage of success rates for the six scenarios are $76.67 \%, 91.67 \%, 28.33 \%, 13.33 \%, 60 \%$, and $53.33 \%$ for scenario 1 to 6 respectively shown in Table 3 (Fakhruzzaman, Riksakomara, and Suryotrisongko 2015).

In the experiment for detecting EEG signal based emotion using deep learning network with principal component-based covariate shift adaptation, the efficiency was evaluated using four experimental steps. In the first step, the emotion was recognized using a deep learning network with a hundred concealed nodes in each layer (DLN100). The feature extraction was used to calculate all of the input features from EEG signals based on DLN. In the second step, the number of concealed nodes has concentrated to 50 (DLN-50) for analyzing the effects of hidden node size in the DLN. In the third experimental step, the PCA was used to lessen the over-fitting problems based on DLN. The last experimental step, the efficiency of the emotion recognition system has enhanced by applying the covariate shift adaptation (CSA) concept to solve the problem of nonstationary for EEG signals. The DLN-100 supported to get the accuracy of $49.52 \%$ for the valence and $46.03 \%$ for the arousal dimension. The 
accuracy slightly decreased to $47.87 \%$ and $45.50 \%$ for DLN-50 shown in Table 4 . The PCA+CSA setup improved the accuracy by $5.55 \%$ and $6.53 \%$ for valence and arousal dimension respectively. The classification accuracy for the DLN with PCA+CSA is $53.42 \%$ and $52.05 \%$ shown in Table 4 (Jirayucharoensak, Pan-Ngum, and Israsena 2014).

Table 3. Success Rate for the Different Testing Scenario with SVM Classifier

\begin{tabular}{ccc}
\hline $\begin{array}{c}\text { Scenario } \\
\text { Number }\end{array}$ & Testing Scenario & $\begin{array}{c}\text { Success Rate (\%) with SVM } \\
\text { Classifier }\end{array}$ \\
\hline $\mathbf{1}$ & Movement of Left Hand & 76.67 \\
$\mathbf{2}$ & Movement of Right Foot & 91.67 \\
$\mathbf{3}$ & Movement of Left Hand and Right & 28.33 \\
$\mathbf{4}$ & Hand Simultaneously \\
& $\begin{array}{c}\text { Movement of Left Hand and Head } \\
\text { Nodding Simultaneously }\end{array}$ & 13.33 \\
$\mathbf{6}$ & $\begin{array}{c}\text { Movement of Left Foot and Right } \\
\text { Foot Simultaneously }\end{array}$ & 60.00 \\
& Movement of Left Foot and Head & 53.33 \\
\hline
\end{tabular}

Table 4. Average Accuracy Results for Arousal and Valence Dimension with Different Methods

\begin{tabular}{ccc}
\hline Method & $\begin{array}{c}\text { Accuracy } \\
(\%) \text { for } \\
\text { Arousal }\end{array}$ & $\begin{array}{c}\text { Accuracy (\%) } \\
\text { for Valence }\end{array}$ \\
\hline DLN-100 & 46.03 & 49.52 \\
DLN-50 & 45.50 & 47.87 \\
PCA & 50.88 & 48.64 \\
PCA+CSA & 53.42 & 52.05 \\
\hline
\end{tabular}

In the above review based on experiment, we observe that, in most cases, the researcher focused on the signal acquisition, processing, and classification. In the classification level, SVM classifier was mostly used to classify the data, but we have required an improved technique which can help to improve the brain activities.

\section{OBSERVATION AND DISCUSSION}

We observe from the human brain sensor activities related to previous research, in most cases, the EEG signal was acquired using a noninvasive acquisition technique from the human brain. After signal acquisition, the data was recorded and preprocessed. After preprocessing, the feature extraction was completed to reduce the noise, artifacts and unwanted signal data. Brain-Vision System using 18 electrodes was performed 
better for recording the signal data in previous research. ICA and PCA have mostly used in case of preprocessing and feature extraction. In the signal classification level SVM, k-NN, QDA, NB, NN contributed for getting the better performance, but SVM was used to classify the data in most cases. Hjorth parameter was highly used to convert the EEG signal into the time-frequency domain. But, it is the fact that, there is no improvement method which can develop the human brain sensor activities. For that, in the future, we will demonstrate a proposed improvement method to improve the human brain activities.

From the above observation, we decide that non-invasive EEG signal achievement method will be the better option to acquire the signal data. ICA can be used to lessen artifacts and noise from the signal data. Fast Fourier Transformation can be considered to reduce poor signal quality. In the future, we want to establish an efficient method which can help to improve the sensor activities of the human brain. By using this efficient method at first, we can acquire the EEG based brain signal data from the selected individual subjects. For acquiring the signal, we can use a proposed neuroheadset device which can contribute to acquire, modify and process the signal data in the human brain swiftly and frequently. Here, we can use Brain-Vision System using 18 electrodes to record the signal data. After recording the signal data, ICA and PCA can be used in case of preprocessing and feature extraction respectively. After that, we can establish a method, with the help of artificial intelligence, which can modify the brain signal into desired improve signal and finally this improved signal will help to develop the human brain activity with the aid of a proposed improvement approach. The overall methodology has been demonstrated to improve the brain activities in Table 5. The overall process can proceed in the proposed neuroheadset device helps to improve the human brain sensor activities. Several iterations may require at the experiment level. The whole process will stop when the acquired signal match or nearly close to the desired improve signal. Hopefully, this will be the greatest discoveries in science and will also be the greatest advancement in the field of artificial intelligence.

Table 5. Overall Methodology for Human Brain Activities Improvement

\begin{tabular}{ccc}
\hline Phase of Methodology & Methods & $\begin{array}{c}\text { Purpose for the Each Phase } \\
\text { of Methodology }\end{array}$ \\
\hline Signal Acquisition & $\begin{array}{c}\text { Non- Invasive Signal } \\
\text { Acquisition }\end{array}$ & $\begin{array}{c}\text { Acquiring the Human } \\
\text { Brain Signal. }\end{array}$ \\
Signal Preprocessing & Independent Component \\
Analysis & $\begin{array}{c}\text { Reduce Noise and Artifacts } \\
\text { from Signal. }\end{array}$ \\
Signal Feature Extraction & Fast Fourier & Extract the Data of Poor \\
& Transformation & Signal Quality \\
Signal Improvement & Proposed Improvement & Improve the Brain Signal. \\
& Method & \\
Human Brain Activities & Proposed Approach & Improve the Human Brain \\
Improvement & & Activities. \\
\hline
\end{tabular}




\section{CONCLUSION}

The main reason for this research is to introduce an efficient method by presenting a critical analysis of the human brain sensor activities related to previous research. This research demonstrated the investigation of brain sensor activities related to previous research based on methods, framework and also experimental analysis to constitute an efficient method. This efficient method will be the combination of all methods which will be required to perform for improving the human brain sensor activities. From the comprehensive analysis, we observe that non-invasive EEG based signal acquisition technique was mostly used to acquire the brain signal data. Independent Component Analysis was highly considered to pre-process the signal data. Fast Fourier Transformation was highly recommended to extract the signal features from the brain signal data. From this analysis, we have demonstrated, that inspect of more classification techniques, there are no methods exist for improving the brain signal and also for developing brain activities. For this, in future research, we will discuss a proposed improvement method for developing the brain signal and will also discuss a proposed approach for developing brain activities. The details framework will be discussed to develop the human brain activities in future research. In future research, the design of a proposed neuroheadset device will also be introduced to develop the human brain activities by executing an efficient method. Due to the huge challenge to control and improve the brain sensor activities of the human brain, this comprehensive analysis will keep a contribution to establish an efficient method proceeds in a proposed neuroheadset device help to receive, process, improve the human brain signal data and also develop the human brain signal activities very swiftly and frequently.

\section{ACKNOWLEDGEMENT}

This research immensely supported through knowledge sharing from ABERT Research Team (AIUB Brain Engineering Research Team) in the Department of Computer Science, American International University - Bangladesh (AIUB).

\section{REFERENCES}

Suzuki, S., Suzuki, Y., Baba, Y., \& Furuta, K. (2005, 6-10 Nov. 2005). Human skill and brain activity in machine operation. 31st Annual Conference of IEEE Industrial Electronics Society, 2005. IECON 2005.

Shuangquan, W., Jie, Y., Ningjiang, C., Xin, C., \& Qinfeng, Z. (2005, 13-15 Oct. 2005). Human activity recognition with user-free accelerometers in the sensor networks. International Conference on Neural Networks and Brain.

Taruno, W. P., Baidillah, M. R., Sulaiman, R. I., Ihsan, M. F., Fatmi, S. E., Muhtadi, A. H., . . . Aljohani, M. (2013, 7-11 April 2013). 4D brain activity scanner using Electrical Capacitance Volume Tomography (ECVT). IEEE 10th International Symposium on Biomedical Imaging. 
Wijayasekara, D., \& Manic, M. (2013, 6-8 June 2013). Human machine interaction via brain activity monitoring. 6th International Conference on Human System Interactions (HSI).

Angotzi, G. N., Baranauskas, G., Vato, A., Bonfanti, A., Zambra, G., Maggiolini, E., . . . Fadiga, L. (2015). A Compact and Autoclavable System for Acute Extracellular Neural Recording and Brain Pressure Monitoring for Humans. IEEE Transactions on Biomedical Circuits and Systems, 9(1), 50-59. doi:10.1109/tbcas.2014.2312794

Boughariou, J., Jallouli, N., Zouch, W., Slima, M. B., \& Hamida, A. B. (2015). Spatial Resolution Improvement of EEG Source Reconstruction Using swLORETA. IEEE Transactions on NanoBioscience, 14(7), 734-739. doi:10.1109/tnb.2015.2477247.

Bastani, K., Kim, S., Z, Nussbaum, M. A., \& Huang, W. (2016). Online Classification and Sensor Selection Optimization With Applications to Human Material Handling Tasks Using Wearable Sensing Technologies. IEEE Transactions on Human-Machine Systems, 46(4), 485-497. doi:10.1109/thms.2016.2537747

Mehmood, R. M., Du, R., \& Lee, H. J. (2017). Optimal Feature Selection and Deep Learning Ensembles Method for Emotion Recognition From Human Brain EEG Sensors. IEEE Access, 5, 14797-14806. doi:10.1109/access.2017.2724555.

Li, C., Yuan, H., Urbano, D., Cha, Y. H., \& Ding, L. (2017, 11-15 July 2017). ICA on sensor or source data: A comparison study in deriving resting state networks from EEG. 39th Annual International Conference of the IEEE Engineering in Medicine and Biology Society (EMBC).

Rushambwa, M. C., \& Mythili, A. (2017, 16-18 March 2017). Impact assessment of mental subliminal activities on the human brain through neuro feedback analysis. Third International Conference on Biosignals, Images and Instrumentation (ICBSII).

Even-Chen, N., Stavisky, S. D., Pandarinath, C., Nuyujukian, P., Blabe, C. H., Hochberg, L. R., . . . Shenoy, K. V. (2018). Feasibility of Automatic Error Detectand-Undo System in Human Intracortical rain(2013);Computer Interfaces. IEEE Transactions on Biomedical Engineering, 65(8), 1771-1784. doi:10.1109/tbme.2017.2776204 
Besio, W. (2017, Oct. 29 2017-Nov. 1 2017). Live demonstration: High-fidelity brain electrical activity from automatic noise cancelling tripolar concentric ring electrode sensor. IEEE SENSORS.

Hebbar, A. (2017, 3-5 Nov. 2017). Augmented intelligence: Enhancing human capabilities. Third International Conference on Research in Computational Intelligence and Communication Networks (ICRCICN).

Turnip, A., Suhendra, M. A., Esti, K. D., \& Sihombing, P. (2017, 9-11 Aug. 2017). Design of extraction method of SSVEP brain activity with IIR Chebyshev. 5th International Conference on Instrumentation, Control, and Automation (ICA).

Rao, T. K., Lakshmi, M. R., \& Prasad, T. (2012). An exploration on brain computer interface and its recent trends. arXiv preprint arXiv:1211.2737.

Mehmood, R. M., \& Lee, H. J. (2015). EEG based emotion recognition from human brain using hjorth parameters and SVM. Int. J. Bio-Sci. Bio-Technol, 7(3), 23-32.

Atkinson, J., \& Campos, D. (2016). Improving BCI-based emotion recognition by combining EEG feature selection and kernel classifiers. Expert Systems with Applications, 47, 35-41.

Gutmann, B., Mierau, A., Hülsdünker, T., Hildebrand, C., Przyklenk, A., Hollmann, W., \& Strüder, H. K. (2015). Effects of physical exercise on individual resting state EEG alpha peak frequency. Neural plasticity, 2015.

Marathe, A. R., Lawhern, V. J., Wu, D., Slayback, D., \& Lance, B. J. (2016). Improved neural signal classification in a rapid serial visual presentation task using active learning. IEEE Transactions on Neural Systems and Rehabilitation Engineering, 24(3), 333-343.

Kao, J. C., Nuyujukian, P., Ryu, S. I., \& Shenoy, K. V. (2017). A high-performance neural prosthesis incorporating discrete state selection with hidden Markov models. IEEE Transactions on Biomedical Engineering, 64(4), 935-945.

Fakhruzzaman, M. N., Riksakomara, E., \& Suryotrisongko, H. (2015). EEG wave identification in human brain with Emotiv EPOC for motor imagery. Procedia Computer Science, 72, 269-276. 
Lakshmi, M. R., Prasad, D. T., \& Prakash, D. V. C. (2014). Survey on EEG signal processing methods. International Journal of Advanced Research in Computer Science and Software Engineering, 4(1).

Jangraw, D. C., Wang, J., Lance, B. J., Chang, S.-F., \& Sajda, P. (2014). Neurally and ocularly informed graph-based models for searching 3D environments. Journal of neural engineering, 11(4), 046003.

Jirayucharoensak, S., Pan-Ngum, S., \& Israsena, P. (2014). EEG-based emotion recognition using deep learning network with principal component based covariate shift adaptation. The Scientific World Journal, 2014.

Sulaiman, N., Cheng Chee, H., Hadi, A. A., Mustafa, M., \& Jadin, S. (2014, 28-30 Nov. 2014). Interpretation of human thought using EEG signals and LabVIEW. IEEE International Conference on Control System, Computing and Engineering (ICCSCE 2014).

Arman, S. I., Ahmed, A., \& Syed, A. (2012). Cost-effective eeg signal acquisition and recording system. International Journal of Bioscience, Biochemistry and Bioinformatics, 2(5), 301.

Karamizadeh, S., Abdullah, S. M., Manaf, A. A., Zamani, M., \& Hooman, A. (2013). An overview of principal component analysis. Journal of Signal and Information Processing, 4(03), 173. 